Acta Crystallographica Section B

Structural

Science

ISSN 0108-7681

\title{
The crystal structure of sorensenite, $\mathrm{Na}_{4} \mathrm{SnBe}_{2}\left(\mathrm{Si}_{3} \mathrm{O}_{9}\right)_{2} \cdot 2 \mathrm{H}_{2} \mathrm{O}$
}

J. Metcalf-Johansen and R. G. Hazell

This electronic document was scanned from an archival copy of material deposited to accompany a paper published in an IUCr journal. In many cases the only accessible copy was a microfilm of a poor-quality original. 


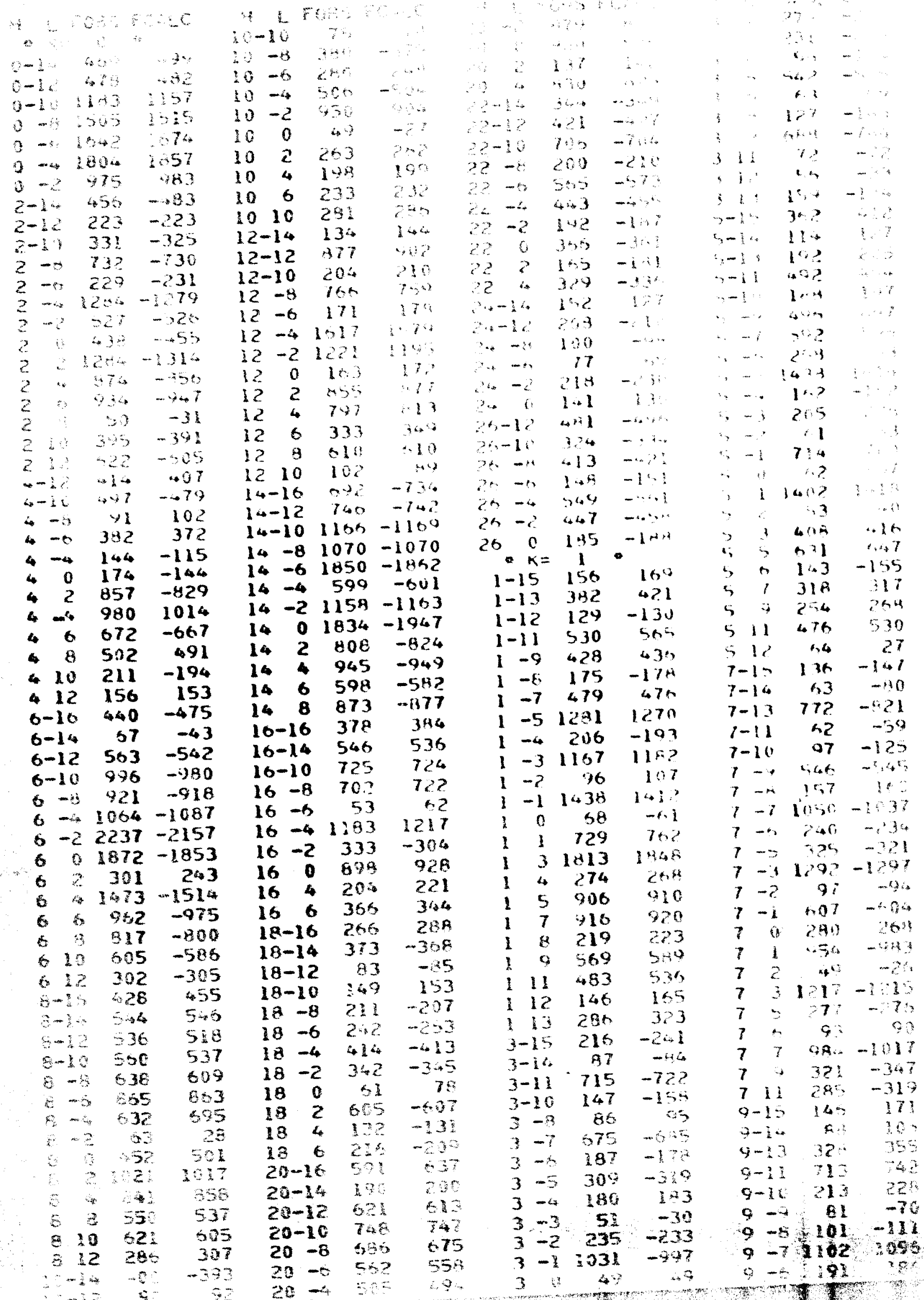




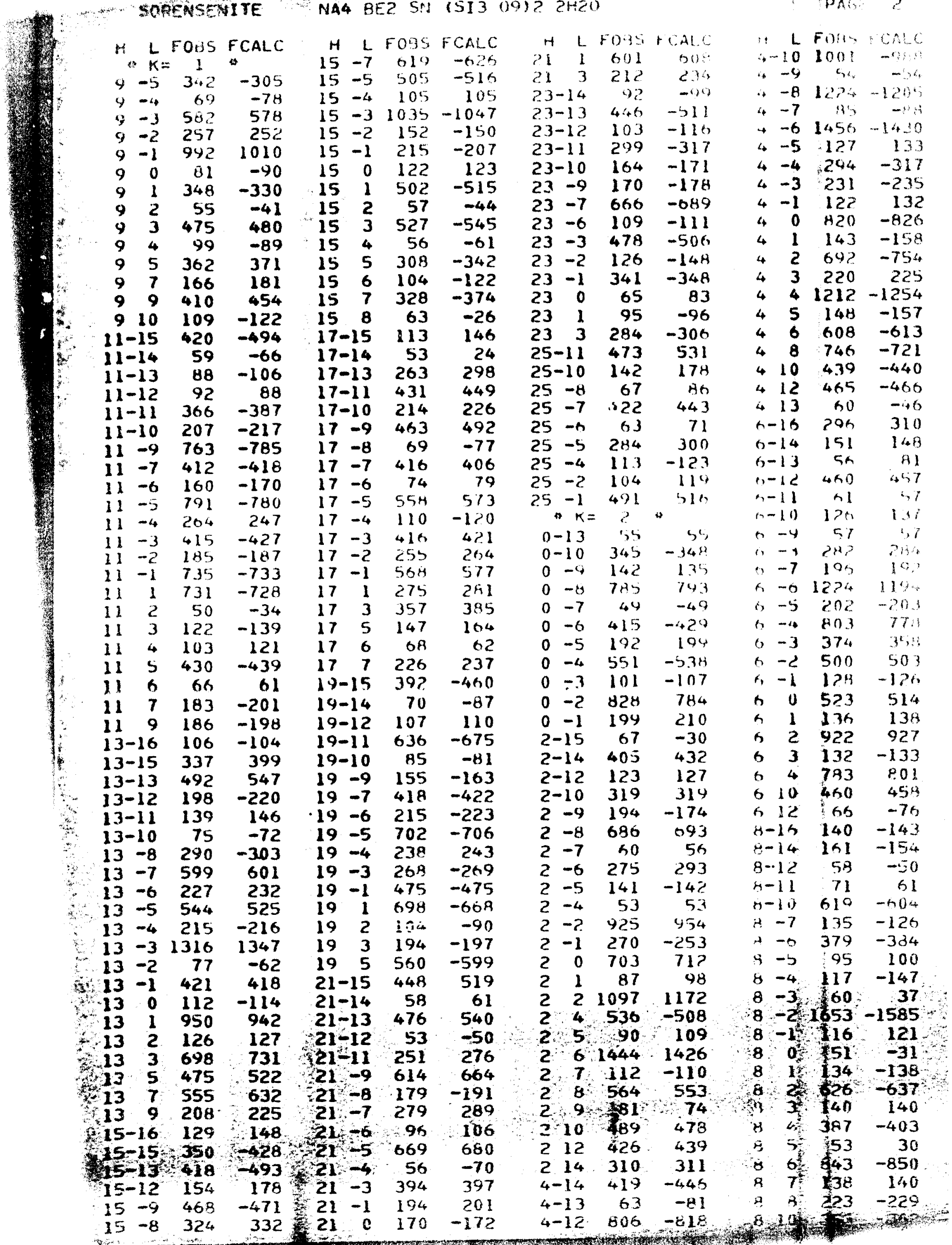




\section{SOLSENITS}

H L FOBS FCALC

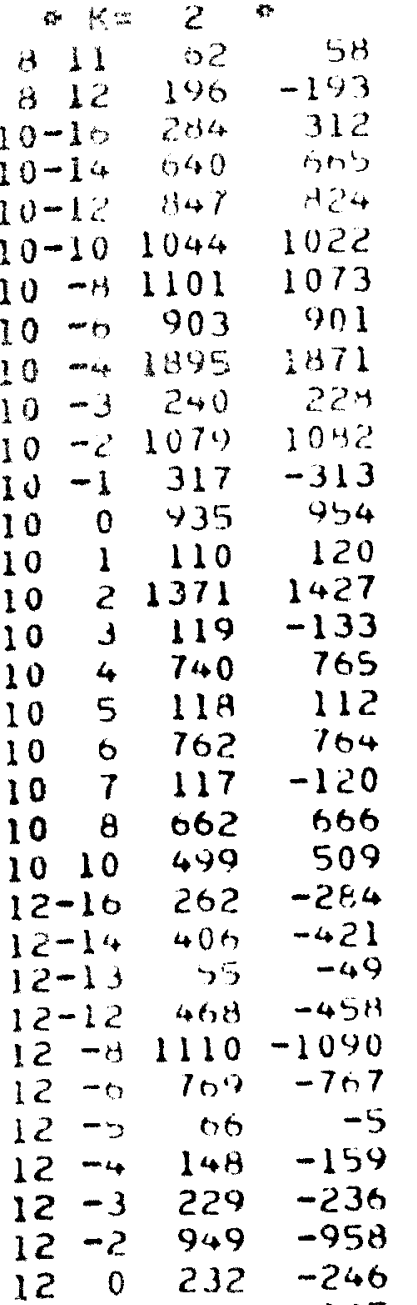

$121104-105$

$1221249-1315$

$\begin{array}{llll}12 & 3 & 51 & 64 \\ 12 & 4 & 60 & 70\end{array}$

$\begin{array}{llll}12 & 4 & 60 & -85\end{array}$

$12 \quad 6 \quad 324 \quad-328$

128

1210

487

142

$14-16$

$14-15$

99

$14-14$

$14-13$

$14-12$

$14-11$

$14-10$

$14-7 \quad 96$

$14-6 \quad 275$

$14-5 \quad 161$

$14-4 \quad 372-360$

$14-2$

140

14. 1137

142.255

143119

14) 4315

14567

14

$14 y$

$16-10$

313

$-65$

08
233

$-307$

NA4 BEZ SA (SIJ 09)? $2 H 2 O$

H L: FOAS FCALC

4 L FUAS FCALC.

$16-12 \quad 227-23 ?$

$16-11 \quad 100$

$: 6-10$

$16-9$

is -8

752

$-6 \quad 472$

$16-4$ 40.3 - 463

$\begin{array}{llll}16 & -3 & 93 & 98\end{array}$

$16-1 \quad 145 \quad 143$

$16 \quad 0 \quad 832 \quad-975$

$16-10 \quad 693$

$501-512$

$94 \quad-107$

$594 \quad-594$ $\begin{array}{rrrr}24 & -8 & 548 & -54 \\ 24 & -7 & 65 & -55 \\ 24 & -6 & 400 & -404 \\ 24 & -5 & 121 & -111 \\ 24 & -4 & 455 & -494 \\ 24 & -2 & 712 & -147 \\ 24 & 0 & 531 & -0101\end{array}$

$\begin{array}{cl}k= & 3 \\ 1-13 & 56\end{array}$

$1-12 \quad 0.7 \quad-6.7$

$\begin{array}{rrr}-11 & 53 & -24\end{array}$

$\begin{array}{llll}1 & -4 & 203 & -603 \\ 1 & -4 & 190 & -60 h\end{array}$

$1-7 \quad 122-114$

$\begin{array}{rrrr}1 & -4 & 41 & 14 \\ 1 & -3 & 2.45 & 24 h\end{array}$

$\begin{array}{rrrr}1 & -2 & 134 & -143\end{array}$

$\begin{array}{rrrr}1 & 0 & 33 & 50 \\ 1 & 1 & 230 & -307\end{array}$

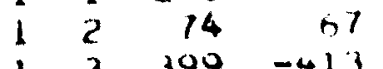

$\begin{array}{lllr}1 & 3 & 399 & -413 \\ 1 & 4 & 191 & 142\end{array}$

$15103-114$

$\begin{array}{rrrr}1 & 5 & 90 & 41 \\ 1 & 7 & 1.31 & -1 \%\end{array}$

$1 \mathrm{H} 73$

$\begin{array}{rr}14 & 453 \\ 1 & 10 \quad 106\end{array}$

111160

112111

113223

$3-15$

372

$\begin{array}{ll}3-14 & 111 \\ 3-13 & 631\end{array}$

$3-11$ कh

$3-10$ 16H

$\begin{array}{lll}3 & -7 & 833\end{array}$

$\begin{array}{lll}3 & -6 & 127\end{array}$

$\begin{array}{lll}3 & -5 & 1749\end{array}$

$3-4 \cdot 123$

$3-3 \quad 521$

$3-1>14$

3

146
497

$\begin{array}{rrr}3 & 1 & 497 \\ 3 & 2 & 34\end{array}$

$\begin{array}{lll}3 & 3 & 1431\end{array}$

$\begin{array}{lll}3 & 4 & 78\end{array}$

7
$-4+30$

4is

$-1+1$

$-720$

140

क.

HCI

$-165$

307

42.3

$-119$

1754

$-124$

540

762

$-155$

532

$-19$

351073

145

$3690-117$

$\begin{array}{llll}3 & 7 & 125 & 100\end{array}$

$\begin{array}{rrrr}3 & 9 & 492 & 434 \\ 3 & 10 & 5 n & 53\end{array}$

$\begin{array}{llll}3 & 10 & 5 n & 516\end{array}$

312

57

313

450

$-45$

$5-15 \quad 462 \quad-489$

$5-13 \quad 391 \quad-346$

$5-12 \quad 62 \quad-42$

$5-11-89-723$

$5-7 \quad 503 \quad-527$

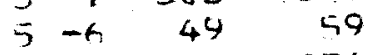

$5-51350-1376$
$1-6 \quad 114 \quad 10 \%$

$-13.1$ in 1

162

164

165

166

167

16
$18-16$

$18-14$

$18-13$

$18-12 \quad 4 H 1 \quad 480$

$14-11$

$1 \mathrm{H}-\mathrm{B} \quad 1175$

$18-6501$

$1 A-5 \quad 114$

$1 H-4 \quad 5 H 7$

$1 H-211.5$

IH 0419

142799

184221

185110

180587

20-15

$20-12$

20-10

$20-9$

$20-8$

$20-7$

$20-6$

$20-5$

$20-3$

$20-2$

202

$20 \quad 3$

204

$22-14$
$22-12$

$22-11$
$22-10$

$22-10$

$\begin{array}{ll}22 & -8 \\ 22 & -7\end{array}$

$\begin{array}{ll}22 & -7 \\ 22 & -6 \\ 22 & -5\end{array}$

$\begin{array}{ll}22 & -5 \\ 22 & -4\end{array}$

$22-3$

$22-2$

220

$22 \cdot 2$

$24-14$

24-13

$24-12$

$24-10$

$-10 A$

$-54 h$
-52

$-46$

75

$-174$

426

579

109

70

696

1172

$50 \%$

117

1210

427

HUS

$22 ?$

111

010

41
$7+4$

$\begin{array}{rr}391 & -364 \\ 63 & -69\end{array}$

$61-51$

80 HI

$58 \quad 66$

$476-500$

$68 \quad 88$

$64-87$

$127-131$

$97-102$

$\begin{array}{rr}81 & 80 \\ 297 & -313\end{array}$

$179 \quad 174$

$354 \quad 339$

$\begin{array}{rr}94 & -76 \\ 549 & 547\end{array}$

547
351

$\begin{array}{rr}85 & 90 \\ 479 & 489\end{array}$

$118-125$

$374 \quad 375$

$81-91$

413415

$490 \quad 494$

163.162
H l FoAS FCAlo

$\begin{array}{lll}5-4 & 217-30 \\ \therefore-4 & 054-14\end{array}$

4 ... 34

$\therefore-11315-11.0$

, i) 1112

$\because \quad 1214-119$

$+3106$

5454

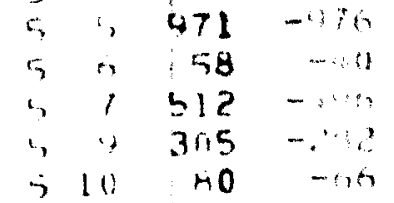

$\begin{array}{llll}\rightarrow & 11 & 100 & -106 \\ 5 & 11 & 106 & -1.63\end{array}$

$7-15 \quad 240-191$

$7-14 \div 56-15$

$7-11 \quad 442 \quad 490$

$7-10 \quad$ क 57

$7-113.24-306$

$7-10 \quad M 4 \quad-2 k$

$\begin{array}{lll}1-4 & 511 & 1.24 \\ 1-4 & 147 & 127\end{array}$

$7-M \quad 147$

$7-1 \quad|H|$ iी

$7 \rightarrow 213-21$

$\begin{array}{llll}7 & -1 & 04 & 153\end{array}$

$\begin{array}{llll}7-1 & \text { ma } & \text { a.t? } \\ 7-1 & \text { mag and }\end{array}$

$\begin{array}{rrr}7 & 78 & \text { in } \\ 7 & 1 & 415\end{array}$

$7.700 \quad 715$

$7 \quad 104-174$

$\begin{array}{rrrr}7 & 0 & 71 & 10 \\ 1 & 1 & n+7 & \cdots 7\end{array}$

$74121 \quad 114$

711131 in

$4-1 . \quad 54 \quad 50$

$\begin{array}{rrr}y-13 & 380 & -347 \\ y-12 & 54 & 57\end{array}$

$y-11: 536 \quad-519$

$4-10 \quad 131 \quad 130$

$9-4 \quad 14 ?-145$

$9-6 \quad 199 \quad 207$

$9-51594 \quad-607$

$4-3$ /391 -385

$9-2$ 1210 207

(1) $-11164-1105$

9 $1 \quad 745 \quad-737$

$\rightarrow=83-45$

$4 \quad 1079-1072$

$\because 4117 \quad 113$

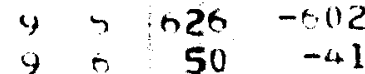

$97 / 318-295$

$941955-839$

$911+20+-190$

$11-1, \quad 67 \quad 69$

$11-10+144-140$

$11-130 \quad 127$

$11-7$ 362 316

$11-6,67-50$

$11-16151588$

$11-4120$ in 


$$
\begin{aligned}
& H \text { L POAS FCALE H L FOAS FCALC } \\
& k=3 \text { in } 17 \text { 2 } 50 \text { o }
\end{aligned}
$$

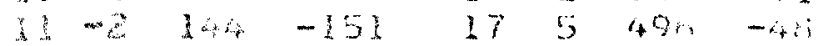

$$
\begin{aligned}
& 12+1296 \quad 1 \% 48 \quad 17 n \text { ht } \quad 0
\end{aligned}
$$

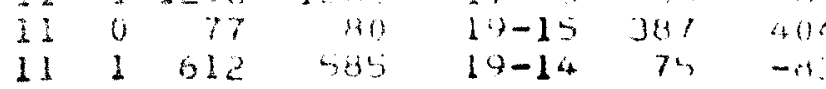

$$
\begin{aligned}
& 112 \begin{array}{lllll}
414 & 45 & 14-13 & 211 & 067
\end{array}
\end{aligned}
$$

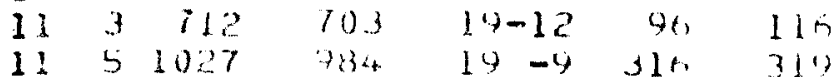

$$
\begin{aligned}
& 11 \quad 7543 \quad 510 \quad 19-7 \quad 167 \quad 174 \\
& 119201 \text { 2as ly }-5 \text { 406 री } \\
& \begin{array}{lllllll}
13-10 & 114 & -117 & 14 & -4 & 120 & 122
\end{array} \\
& \begin{array}{rrrrrr}
13-15 & 57 & -23 & 19 & -3 & 50 \\
13-13 & 203 & -209 & 19 & -1 & 560
\end{array} \\
& \begin{array}{ccccccc}
13-12 & 1.38 & -131 & 19 & 1 & 390 & 37 h
\end{array} \\
& \text { 13-11 } 168 \text { 167 } 193 \text { 111 } 104 \\
& \begin{array}{lllllll}
13-10 & 111 & -134 & 19 & 4 & 121 & 119 \\
13-9 & 415 & -430 & 14 & 5 & 420 & 146
\end{array} \\
& \begin{array}{llllll}
13-8 & 97 & -98 & 21-15 & 190 & -200
\end{array} \\
& \begin{array}{llllll}
13-7 & 286 & 28 H & 21-13 & 102 & -153
\end{array}
\end{aligned}
$$

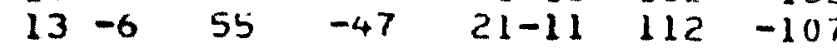

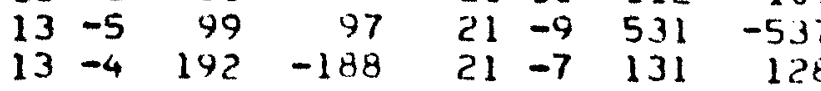

$$
\begin{aligned}
& \begin{array}{lllllll}
13 & -3 & 506 & -508 & 21 & -0 & 61
\end{array}
\end{aligned}
$$

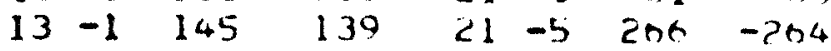

$$
\begin{aligned}
& \begin{array}{llllllll}
13 & 0 & 157 & -157 & 21 & -3 & 18 \% & -144
\end{array} \\
& \begin{array}{rrrrrrrr}
13 & 1 & 304 & -297 & 21 & -2 & 55 & 37 \\
13 & 2 & 93 & 40 & 21 & -1 & 41 & -84.3 \\
13 & 3 & 39 & -74 & 21 & 0 & 60 & -64
\end{array} \\
& 13449-37 \text { 21 } 1<4207.7 \\
& \begin{array}{lllllll}
13 & 5 & 90 & -+2 & 2.3-14 & 102 & -40
\end{array} \\
& \begin{array}{lllllll}
13 & 7 & 247 & -229 & 23-11 & 334 & 34 \\
13 & 8 & 07 & 63 & 23-10 & 145 & -139
\end{array}
\end{aligned}
$$

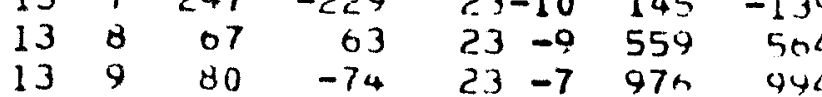

$$
\begin{aligned}
& \begin{array}{lllllll}
15-15 & 153 & 175 & 23 & -6 & 131 & -133
\end{array}
\end{aligned}
$$

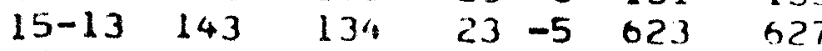

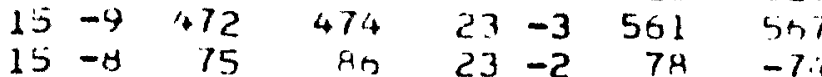

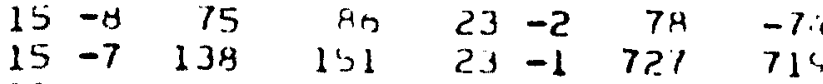

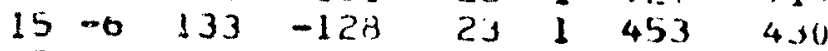

$$
\begin{aligned}
& \begin{array}{llllll}
15-4 & 81 & 43 & 25-13 & 207 & -215
\end{array} \\
& \begin{array}{rrrrrr}
15-2 & 158 & -175 & 25-10 & 99 & 104 \\
15-1 & 218 & 218 & 25-9 & 247 & -257
\end{array}
\end{aligned}
$$

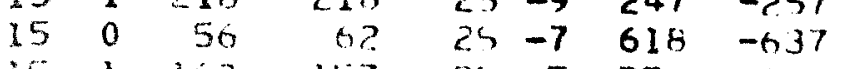

$$
\begin{aligned}
& \begin{array}{llllllll}
15 & 1 & 103 & 157 & 25 & -5 & 554 & -564
\end{array}
\end{aligned}
$$

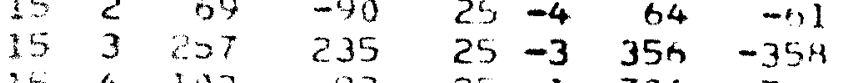

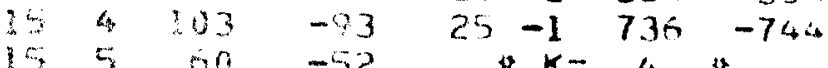

$$
\begin{aligned}
& 15 \quad 7 \quad 354 \quad 321 \quad 0-13 \quad 103 \quad 105
\end{aligned}
$$

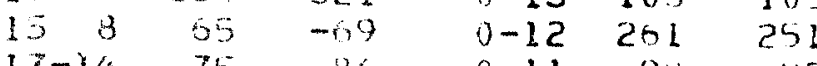

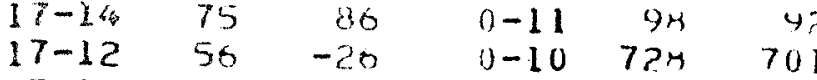

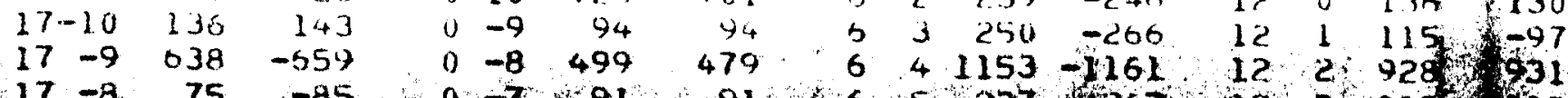

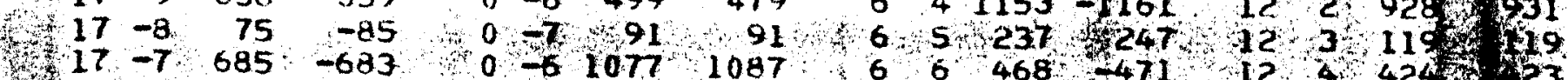

$$
\begin{aligned}
& \begin{array}{lllllllllllllll}
17 & -6 & 98 & 92 & 0 & -5 & 295 & 289 & 6 & 7 & 133 & -128 & 12 & 5 & 205
\end{array}
\end{aligned}
$$

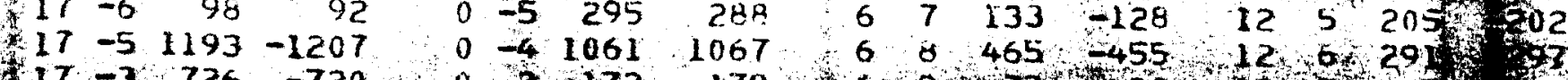

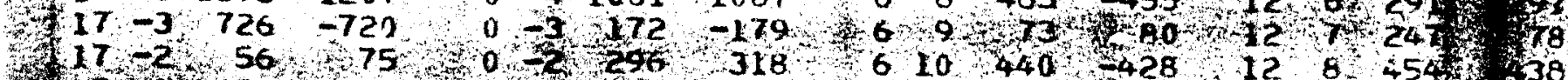

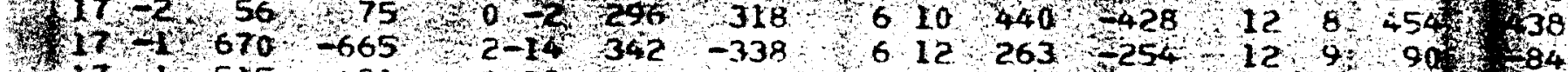

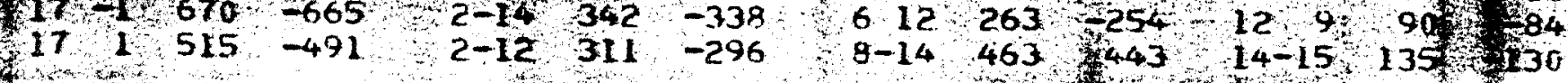

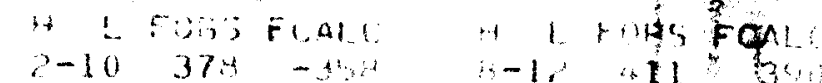

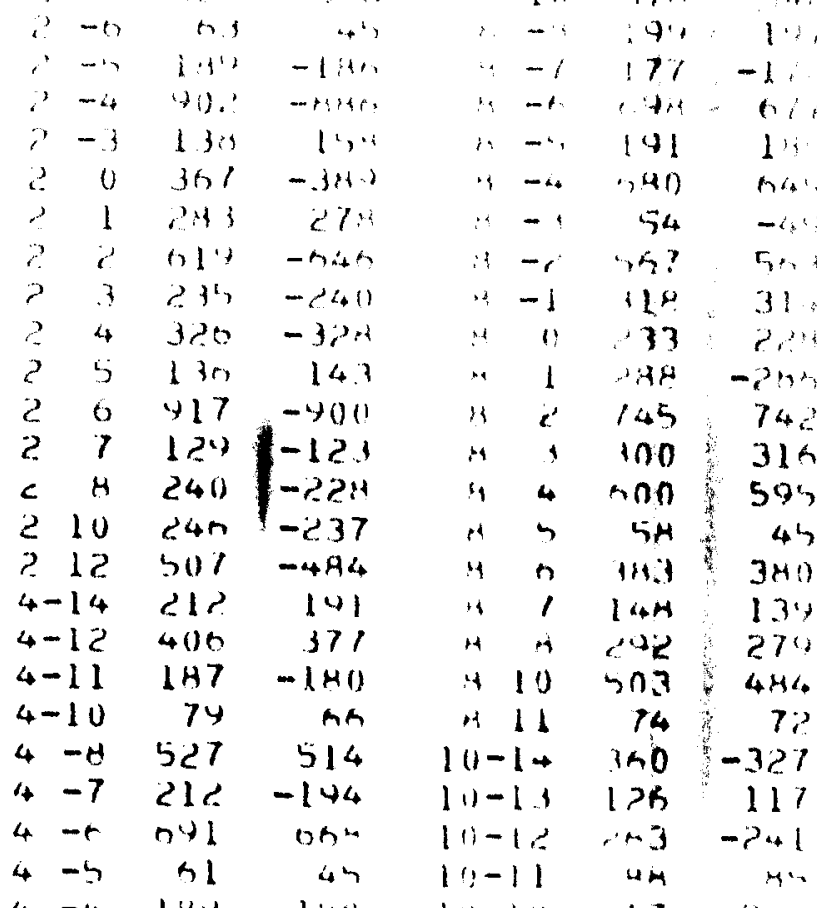

$$
\begin{aligned}
& 4-3.24-119 \quad 11-0 \text { in }-100
\end{aligned}
$$

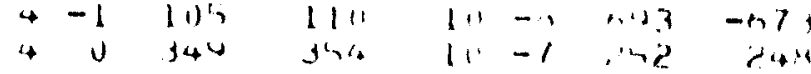

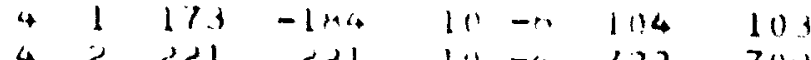

$$
\begin{aligned}
& 43 \text { lin } 1>1 \text { lit }-1 \text { lal } 13 n \\
& 44553 \quad 5 n>110-2 \quad 00-32 \\
& \begin{array}{ll|lllll}
4.0 & 204 & -144 & 11 & 0 & 109 & -523
\end{array}
\end{aligned}
$$

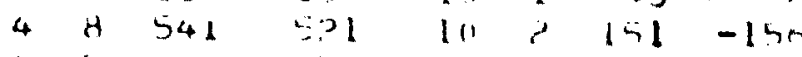

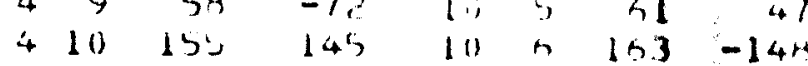

$$
\begin{aligned}
& 412 \quad 174 \quad 177 \quad 11,7 \quad 167-140 \\
& 6-12 \quad 441 \quad-4 h 6 \text { (1) } 10 \text { a6 } 55 \\
& \text { 6-11 } 16 J \text { 146 1P-14 } 149+179
\end{aligned}
$$

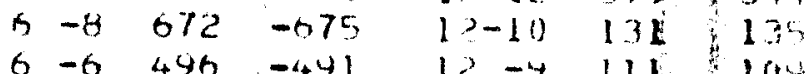

$$
\begin{aligned}
& \begin{array}{llllllll}
6 & -5 & 103 & 105 & 1
\end{array}-4 \quad 700 \quad 670
\end{aligned}
$$

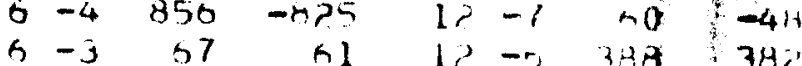

$$
\begin{aligned}
& 3-r 950-916 \quad 1,2-3 \text { ris } 74
\end{aligned}
$$

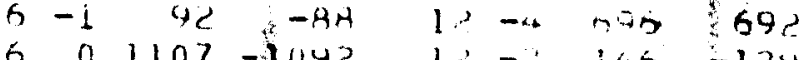

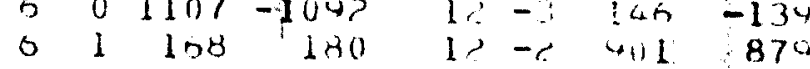

$$
\begin{aligned}
& 62234 \text {-24h le u } 135+130 \\
& \text { b } 3250-266 \quad 12 \quad 1 \quad 115,-97
\end{aligned}
$$




$$
\begin{aligned}
& \text { H L FuHs PCALC } \\
& \begin{array}{c}
k=4 \\
14-14+12,-40
\end{array}
\end{aligned}
$$$$
14-13 \quad 13 i^{2}-13 i
$$$$
14-12>31-13
$$$$
14-11 \quad 171 \quad \cdots 1,4
$$$$
\begin{array}{lll}
1 \rightarrow-10 & 50 / & -49
\end{array}
$$

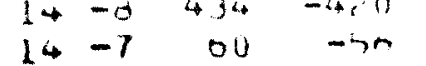$$
14-01111-1101
$$$$
\begin{array}{lll}
14-5 & 150 & -147 \\
14-4 & 103 & -30 !
\end{array}
$$$$
14-4 \text { jo.3 }-301
$$$$
\begin{array}{rrr}
1+-2 & 505 & -404 \\
1+-1 & 34 & -40
\end{array}
$$$$
\begin{array}{rrrr}
1+ & -1 & 34 & -40 \\
14 & 0 & 995 & -974
\end{array}
$$$$
\begin{array}{rrrr}
14 & 1 & 74 & 03 \\
14 & 2 & 375 & -364
\end{array}
$$$$
\begin{array}{llll}
14 & 2 & 375 & -369 \\
14 & 1 & 130 & -139
\end{array}
$$$$
144700-042
$$$$
\begin{array}{rrrr}
14 & 5 & 54 & 41 \\
14 & 0 & 303 & -244
\end{array}
$$$$
147 \text { oर } 33
$$$$
145 \quad 523-563
$$$$
10-14 \quad 535 \quad 501
$$$$
\text { 10-12 179 105 }
$$$$
\text { 16-11 184 } 183
$$$$
\text { 16-10 754 } 720
$$$$
\begin{array}{llll}
16 & -8 & 407 & 454 \\
16 & -6 & 134 & 129
\end{array}
$$$$
\begin{array}{llll}
16 & -4 & 946 & 934
\end{array}
$$$$
\begin{array}{lll}
16 & -1 \quad 157 & 149
\end{array}
$$$$
\begin{array}{rrrr}
16 & 0 & 045 & 034 \\
16 & 1 & 157 & -169
\end{array}
$$$$
\begin{array}{llll}
16 & 2 & 136 & 125
\end{array}
$$$$
\begin{array}{llll}
16 & 4 & 140 & 127
\end{array}
$$$$
160505+\mathrm{H2}
$$$$
18-14 \quad 321-312
$$$$
18-13 \quad 145 \quad 131
$$$$
18-12 \quad 24.3 \quad-243
$$$$
18-10 \text { 78 }-75
$$$$
18-8 \quad 572-500
$$$$
\begin{array}{llll}
18 & -6 & 206 & -198 \\
18 & -4 & 423 & -420
\end{array}
$$$$
\begin{array}{rrrr}
18 & -4 & 423 & -420 \\
18 & -3 & 215 & 214
\end{array}
$$$$
\text { Ib }-2 \text { ibs }-1+37
$$$$
18-1 \quad 120-113
$$$$
180 \text { 13y }-182
$$$$
18175 \quad 71
$$$$
18 \quad 2.577-501
$$$$
18+103-145
$$$$
285104 \text { in }
$$$$
20-14 \quad 170 \text { !7n }
$$$$
20 \cdot 12 \text { 343 381 }
$$$$
\text { 20-11 } 114-110
$$$$
\begin{array}{ccc}
20-10 & 440 & 425
\end{array}
$$

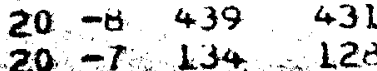$$
20,-5+533-527
$$

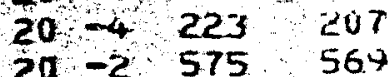$$
20 \quad 0 \quad 554 \quad 542
$$

it L FOHS FCALC

$2 ?-1:$ 505 -49

$$
3-10 \text { 5\% -4, }
$$$$
\text { 2. }-454
$$$$
\therefore-4 \text { cis }
$$$$
\therefore-0 \quad 4+4
$$$$
\because-4,54
$$

$\therefore-1 \quad 103$

$\therefore-2$ <0n -140

$\therefore-1$ मr $-n$

$\begin{array}{rrrr}\therefore & 0 & 3 A n & -111 \\ \therefore & 1 & 147 & 111\end{array}$

$$
\because \quad \therefore \quad 153-1+1
$$$$
4=5
$$

$1-12$ को

$\begin{array}{rr}1-12 & 47 \\ 1-11 & 74 h\end{array}$

$1-4440$

$1-A \quad 161$

$1-7$ HOT

1 -5 1152 114

$1-4 \quad 210$

$1-2<55$

10073

$\begin{array}{lll}1 & 1 & 906 \\ 1 & 2 & 174\end{array}$

131504

14253

15799

17908

$14 \quad 67$

19670

111549

112130

113340

$3-13 \quad 194$

$3-12$

$3-11$

121

363

$3-9$

$3-7$

$3-6$

a1 04

$\begin{array}{ll}275 & -279 \\ 113 & -44\end{array}$

$3-4$

$3-3 \quad 209$

$3-2 \quad 255$

$3-1$ no?

343
$3 \quad 40$

$$
31
$$

32

33

$3416 \%-1 \%$

$35110-132$

$35102-40$

$3 \quad 9 \quad 313-301$

$311 \times 13 \quad 130$

$5-14,75 \quad-52$

$5-13,03 \quad 09$

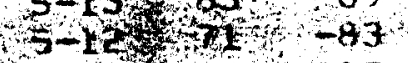

$5-11+320$

$5-95154$

313

$5-8661-66$

$5-7+245-24 d$

$5-6,237,-225$

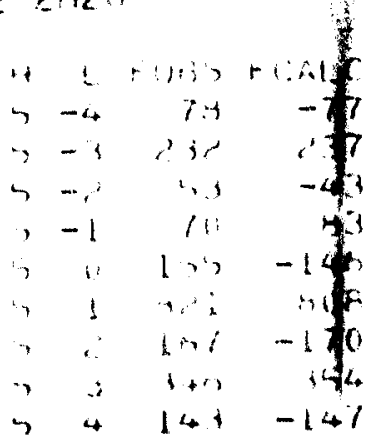

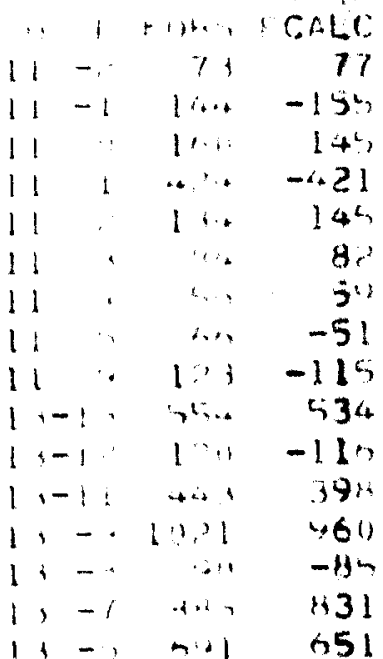

711 गे० 241

$1-1+$ wis -204

$1-11$ oth - ons

7-10 ye 5il

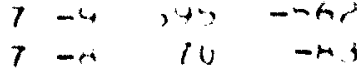

$7-7 \quad 114 i-111+3$

$\begin{array}{lll}7-9 & 400 & -4 k \mu \\ 7-4 & 10.4 & -13\end{array}$

$7-1.114-113$

$7-6 \quad+1 \quad 101$

$1-1$ nol -974

71 प4य - 110

$7 \quad 2 \quad 227-230$

$\begin{array}{rrrr}7 & 3 & 10+0 & -1040 \\ 7 & 4 & 50 & 35\end{array}$

$75342-3 H A$

7 o $64 \quad 44$

$771032-100$

$7+74-54$

$7+424-410$

$4-14$ 12.1 113

$4-13$ l45 134

4.11 6y4 643

$\begin{array}{rrr}7-10 & 35 \\ 9-4 & 134 & -127\end{array}$

$\begin{array}{rrrr}9 & -4 & 13 H & -127 \\ 9 & -4 & 44 & 97\end{array}$

$\begin{array}{lll}9-4 & 44 & 53 \\ 9 & -7 & 54\end{array}$

$\begin{array}{rrr}9 & -7 & 74 \\ 4 & 7 n\end{array}$

$9-5404-407$

$4 \quad-145134$

$9-3+14 \quad 404$

$4-1474$

$$
40 \text { ग0 }
$$

$91,10-510$

$\rightarrow \quad 1 \rightarrow 2190$

$1, \ldots 1,14,-14$.

$11-11411-1324$

$1+-1.11 \quad-104$

i, $-1+11$ - 41

$\begin{array}{llll}11 & 1 & 14 & 144\end{array}$

111 lian 1037

$1:, 7$ i.t $74 \mathrm{~m}$

$1+\ldots \quad-91$

$1:-131171$

$\begin{array}{llll}1 i 1 & 700 & 100 \\ 1-1.0 & 71 & -90\end{array}$

$15-1$. hal $-61 d$

$\mid \zeta-1 \zeta$ HO $\mathrm{AH}$

$|\zeta-1|$ H94 - सह2?

$15-111 \quad 155 \quad-133$

is $\rightarrow 443=480$

$1 ;-9$ Ho -7 ?

is -1 74? -734

$17-9$ क.

is $-12120-115$

$15-1140-1504$

$1:-1$ ili $-29 \pi$

13 1in -144

in: 1 hha $100 \quad-64$

l' 1 hisl -646

$\begin{array}{lll}14 & -14 & -362 \\ 14 & 1 \geqslant 4 & -104\end{array}$

$11-1, \quad 1+4$ I27

$11-11$ :11 KHO

$11-1 . \quad \therefore \quad-5 \%$

$11-\cdots \quad 17, \quad 103$

$17-1$ in 272

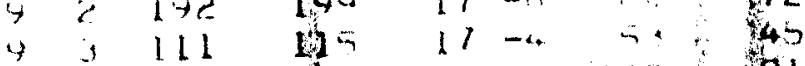

45 144 45 17-, 113 31

$\Rightarrow$ in 124 37 $17-1,341,337$

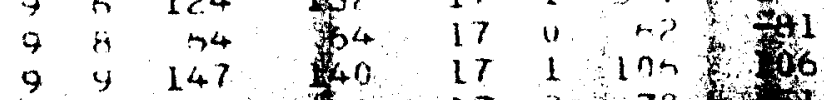

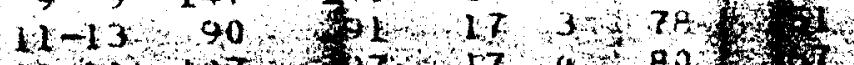

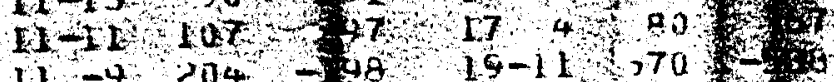

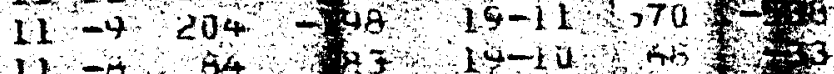

1 $-7,734,25,19-6,109$ $17-6,130+26,10,-2,153$ 


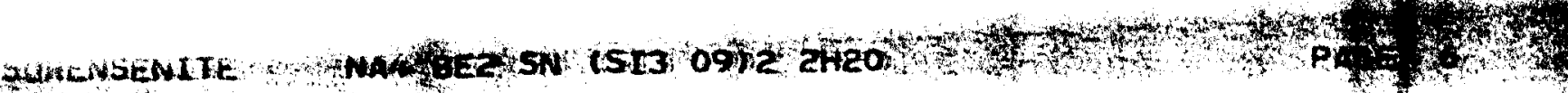

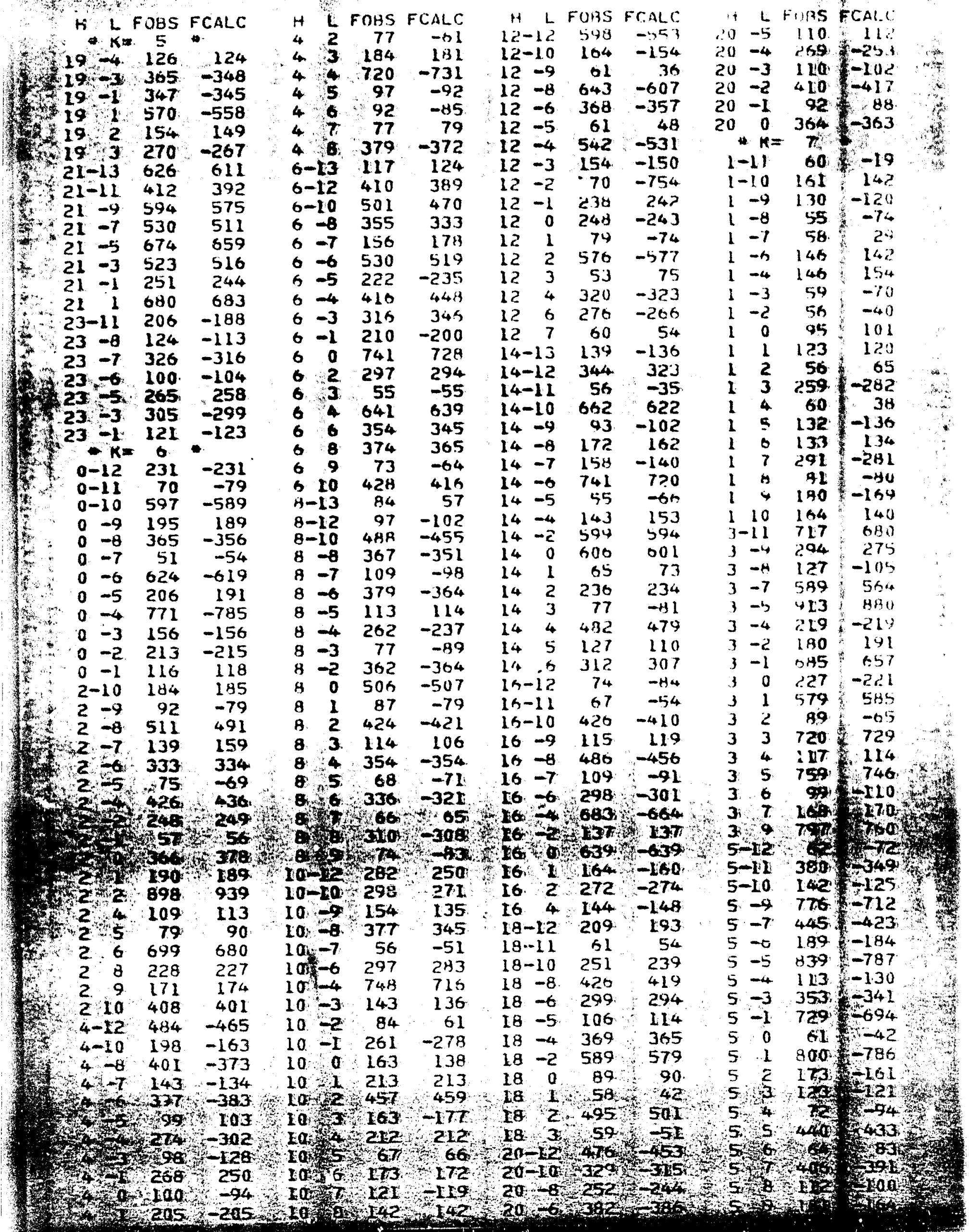


HH L FUGS FCALC $K=T$ :

$7-11 \quad$ IIA -111

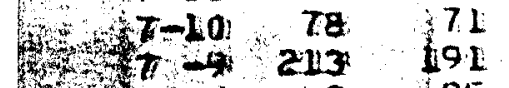

$\begin{array}{rrr}7 \pi & 213 & 49\end{array}$

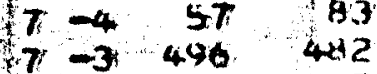

$\begin{array}{rrrr}7 & -3 & 496 & 57\end{array}$

70 4b 05

71143

7 3. 398:

T. 4.69

(7. 7295

$9-1279$

$9-11 \quad 400$

$9-9 \quad 174$

$\begin{array}{lll}9 & -8 & 154 \\ 9 & -7 & 877\end{array}$

90 क 0

$\begin{array}{lll}9 & -5 & 500 \\ 9 & -4 & 30\end{array}$

9. $-3 \quad 315$

9. $-2 \quad 107$

$9-1 \quad 833$

9 $0 \quad 179$

$91404-340$

9 $3685-665$

4 I ITZ ITI

$953+5-3+30$

97 37A -36h

$11-11$ o70 014

II-10 $74 \quad 70$

Il $-9 \quad 910$ H2F

$11-3 \quad 132$ 124.

II $-7 \quad 42 \quad 397$

I1 $0 \quad 139 \quad 139$

Il -4 वा -47

I1 -3671

Il -2 1.10

$11-1 \quad+15$

il 0128

Iil $L$ ISA

जil 2 प5

II 3 3 35

1ิ $4 \quad 54$

II $5 \pi$

II-11 197

I3-10 123 - 112

$\begin{array}{rrr}17 & 280 & -2 \pi 5 \\ 13 & 97 & 99\end{array}$

II -7 IIJ 104

I3 $\rightarrow 181-195$

I3 -5 158 $-15 A$

$\begin{array}{llll}13 & -4 & 71 & -73\end{array}$

$13-3 \quad 124-131$

$13-1 \quad 153 \quad 133$

130 121 -115

$13 \quad 1 \quad 232-25$

[3] $170-547$

$13,4126-124$
$H$ F FUAS FCALC

15 -7 19. L LA

$15-6.109-110$

$15-5 \quad 95 \quad-13 x$

$15-4$ 95. $-7 \mathrm{~h}$

$\begin{array}{ll}15 & -3 \\ 15 & -2 \\ 2525 & -140\end{array}$

$15-10$ 90 $\rightarrow$ ?

150,104

15 il 125

153.124

$15 \quad 4: 104$

IT T-I I

$17-9 \quad 541$

IT -7 प्र

IT -6 \&9

$\begin{array}{lll}17 & -5 & 045 \\ 17 & -4 & 120\end{array}$

$17-3$

$17-2$

$17-11$

174

172

(4) 10

$19-4 \quad 304$

$19-\pi \quad 305$

ly -6 . 300

$14-5.450$

(4) -3

is -2

la -11

Hn

190

374

- $K=$

$0 \rightarrow$

1) $-7 \quad 130$

$10 \quad 193$

$\begin{array}{lll}10 & -5 & \text { III) }\end{array}$

$0-4 \quad 107$

$\begin{array}{lll}0 & -3 & 5.3\end{array}$

1) -2

1) -

$3-1$

$\begin{array}{rr}2+344 & -350 \\ 2-70 & 10 ?\end{array}$

$2-4003 \quad-45$

$2-4063-371$

$2-3$ L4A I44

2. -2 401 -447

? $-12-46 \quad-30$

20 2हn -2क्य

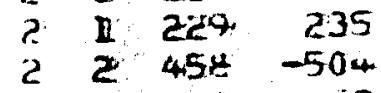

$\begin{array}{llll}2 & 3 & 40 & -52\end{array}$

? 5 91 100

$2 \pi$ 6? 50

c $25 t-255$

2 5 97 of

4-nण 4 45,396 if L Fint Frate

\begin{tabular}{|c|c|c|c|c|c|c|}
\hline & & & & & & \\
\hline$=-1$ & 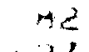 & $=0$ & $1 \%$ & $\because$ & $i \cdots$ & 32 \\
\hline & 421 & & 1, & ! & $\cdots$ & 5 \\
\hline & יAM & מיח כימ & L.' & $\therefore$ & inis & smo \\
\hline 3 & 173 & -74 & ur & 3 & s & j \\
\hline a & 450 & 479 & be & 4 & LTTE & \\
\hline & 254 & 254 & $1 / 4$ & -1 & 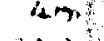 & 4 (1) \\
\hline 7 & 157 & 14011 & $1 *$ & $-\cdot$ & +1.2 & $x$ \\
\hline-10 & 512 & $50 ?$ & 14 & 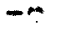 & $\angle 47$ & \\
\hline-10 & $x>3$ & -1.49 & L.t & -4 & los & RII \\
\hline-4 & 12? & -124 & $1 / 4$ & -4 & $4 \times 4$ & 20 \\
\hline$-\theta$ & Itsh & -1.72 & l 4 & -1 & Iny & 115 \\
\hline-7 & Sחו & $-1>1$ & L 4 &,.- & -1 & \\
\hline-3 & 454 & $-4 n ?$ & L/4 & -1 & 74 & -6 \\
\hline ד & ist & Loit & 1. & 1. & 419 & \\
\hline$-\infty$ & 3 & $-20^{\mathrm{m}}$ & 1. & 1 & . & \\
\hline-3 & 1.10 & -1.27 & $1 \cdots$ & 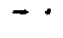 & 1,4 & -1 \\
\hline-6 & $27 \mathrm{~m}$ & $-3 i n$ & $1 \cdot 2$ & - & ${ }^{+} \ldots$ & \\
\hline-1 & inn & $-1(i)$ & 1. & -1 & $\cdot 1$ & $\rightarrow$ \\
\hline 0 & C4+1 & $-6.0 r$ & $1 \cdot$ & $-a$ & f $\ldots$. & 140 \\
\hline ? & RS'ל & $-x_{1}+t_{1}$ & $1 \mathrm{~m}$ & $\rightarrow$ & ") هـ & \\
\hline 3 & $L+1$ & -150 & In & -4 & $\because 2$ & 3 \\
\hline 4 & $41 n$ & -4.30 & In & -4 & $L=L$ & -15 \\
\hline 5 & 1125 & lite & im & $\rightarrow$ & 15,2 & \\
\hline 6 & 126 & $-10)^{2}$ & 17 & -1 & $7 \%$ & -7 \\
\hline$r$ & 120 & - & Lr & " & 41 & 450 \\
\hline
\end{tabular}

$4-11$ मa

$m-10$ 3ns

$4-i$ an

$A-7$

$n-m \quad 214$

$4-5 \quad 44$

$4-4 \quad 241$

$4-3 \quad \ln 4$

$A-? \quad j+0$

$4-1$

$+9$

+1 Lis

if 2 jot

H 3 वा

$4 \quad 47 A$

$+557$

404274

iin $\rightarrow$ 42 $-7 \mathrm{~B}$

$10-4689040$

no $-\pi$ 23A1 255

HO -6 360 - 349

I0) $-4 \quad 698 \quad-777$

10) -2 52' -555

ID -1 ILC 125

$12^{2} 0$ 503k -500

102 h.L -627

$\begin{array}{llll}10 & 9 & 74 & 73 \\ 10 & 4 & 32 & -32 \%\end{array}$

105 ol 5 क

$12-10$ - 50 45

$12-4 \quad 177.174$

L3 -8 400 466

132

mis

a 74

(1)

118

IS

$\$ 7$

$-24$

347

4h

$5 ?$

511

$$
\because 4=0 *
$$

$\cdots \quad \ln 2 \quad 17$

$-1 \quad 471 \quad 443$

- 52 -

- 41 4la

-19 1? 120

-1 i.e. 3h?

$1-6100007$

$1-1.400$

$\begin{array}{lll}11 & 44 & -404 \\ 1 & 407 & 407\end{array}$

$\therefore \quad 77$

1 ine EMS

$1-. \quad I L \quad-7$.

$1-1,711-224$

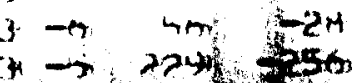

$3-6,760$ क 107

$3-2=45 x+2$

$3-1 \%-3 n \pi x=2 \pi$

$30.53 \pi, 30$

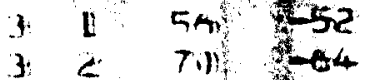

$3 \pm 112 \frac{1131}{31}$

3 - $7 m$ - 8 -

$3=\therefore \div 2 \geq$

$1 \rightarrow 105$ पूसक

$\rightarrow$ and -100

ᄀ. $-7 \%$ \& 74

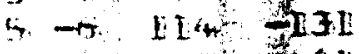

$5-5$ - 45 t

5. $-3 \quad 37430 \%$

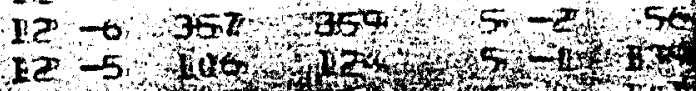


$-2,1 \mathrm{H}$ \%

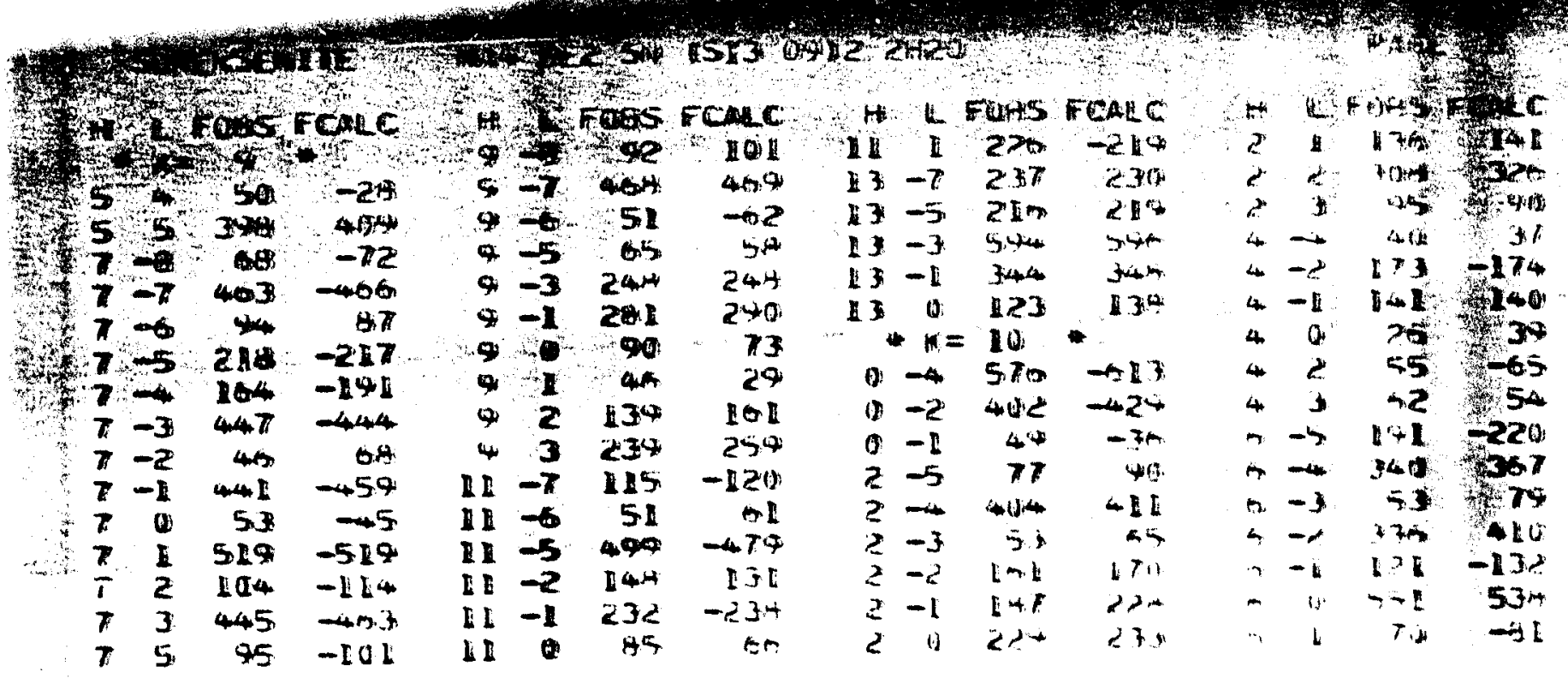


Table $I^{-} I_{\text {est }}$ hke, $\sin ^{2} \theta, F_{c b s}$ and d-spacings for Sorensenite. Guinier de woIf exposure at $20^{\circ} \mathrm{C}$ using Cuka-radiation, $\lambda=1.5418 \lambda$, and NaCl as internal standard.

\begin{tabular}{|c|c|c|c|c|c|c|c|}
\hline Fest & $\mathbf{n}$ & k & $\ell$ & $\sin ^{2} \theta \cos$ & $\sin ^{2} \theta \operatorname{colc}$ & Pobs & $d($ hke $\varepsilon)$ obs \\
\hline 2 & 2 & 0 & 0 & 0.0069 & $9.00 \div 0$ & 86 & 9.2448 \\
\hline 9 & $I$ & $:$ & $\bar{E}$ & 0.0148 & 0.0289 & 283 & 6.3259 \\
\hline$i$ & 2 & 0 & $\bar{z}$ & 0.0166 & $0.0: 67$ & 203 & 5.9651 \\
\hline 2 & & & & analcine & & & $(5.6: 67)$ \\
\hline$x$ & $\left\{\begin{array}{l}I \\
0\end{array}\right.$ & 2 & $\frac{i}{2}$ & 0.0205 & $\begin{array}{l}0.0204 \\
0.0208\end{array}$ & $\left.\begin{array}{l}1 \geq 3 \\
192\end{array}\right\}$ & 5.3793 \\
\hline 4 & 3 & 13 & $\bar{I}$ & 0.0233 & 0.0238 & 203 & 5.0045 \\
\hline 2 & & & & unident 28 & IEne & & \\
\hline$<I$ & 3 & $i$ & $\bar{z}$ & 0.0305 & 0.0307 & 26 & $.004 a$ \\
\hline$<:$ & & & & uniderti: & ifne & & \\
\hline$<:$ & & & & IOW-aitis & Ene & & $(4.0 \times 76)$ \\
\hline 4 & 2 & $\therefore$ & 2 & 0.0386 & 0.0389 & 252 & $3.92 \times 5$ \\
\hline$<:$ & 3 & $:$ & : & 0.0397 & 0.0400 & $53-$ & 3.8600 \\
\hline$<1$ & 0 & 2 & 0 & 0.0426 & 0.0429 & $\$ 1$ & 3.7281 \\
\hline$\Sigma$ & 5 & i & $\bar{I}$ & 0.0457 & 0.0460 & 220 & 3.6016 \\
\hline$<i$ & 0 & 2 & I & 8.047T & 0.0481 & 39 & 3.5243 \\
\hline$\xi$ & $\left\{\begin{array}{l}2 \\
2\end{array}\right.$ & $\begin{array}{l}z \\
z\end{array}$ & $\bar{i}$ & 6.0497 & $\begin{array}{l}0.0495 \\
0.0499\end{array}$ & $\left.\begin{array}{r}53 \\
138\end{array}\right\}$ & $3 . \Delta 503$ \\
\hline 8 & $\left\{\begin{array}{l}6 \\
1\end{array}\right.$ & $\begin{array}{l}0 \\
\text { I }\end{array}$ & $\frac{\overline{2}}{3}$ & c.0506 & $\begin{array}{l}0.0508 \\
0.0509\end{array}$ & $\left.\begin{array}{l}439 \\
229\end{array}\right\}$ & 3.4209 \\
\hline$<<1$ & & & & inidentif & Iine & & \\
\hline$<I$ & & & & low-albit & ine & & $(3.22)$ \\
\hline 5 & 2 & 2 & $\bar{\Sigma}$ & 0.0592 & 0.0596 & 182 & 3.1616 \\
\hline 6 & $\left\{\begin{array}{l}6 \\
0\end{array}\right.$ & $\begin{array}{l}0 \\
2\end{array}$ & $\begin{array}{l}0 \\
2\end{array}$ & 0.0635 & $\begin{array}{l}0.0632 \\
0.0637\end{array}$ & $\left.\begin{array}{l}368 \\
162\end{array}\right\}$ & 3.0529 \\
\hline$\pi$ & $\left\{\begin{array}{l}1 \\
2\end{array}\right.$ & $\frac{1}{a}$ & $\frac{3}{4}$ & 0.0673 & $\begin{array}{l}0.0675 \\
0.0680\end{array}$ & $\left.\begin{array}{l}356 \\
252\end{array}\right\}$ & 2.9660 \\
\hline IO & 4 & 2 & $\bar{\Sigma}$ & 0.0695 & 0.0696 & 286 & 2.9186 \\
\hline 4 & $\{1$ & $\begin{array}{l}0 \\
2\end{array}$ & 2 & 0.0709 & $\begin{array}{l}0.0710 \\
0.0710\end{array}$ & $\left.\begin{array}{l}168 \\
161\end{array}\right\}$ & 2.8910 \\
\hline
\end{tabular}
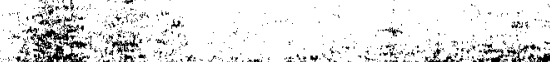


\begin{tabular}{|c|c|c|c|c|c|c|c|}
\hline$I_{\text {est }}$ & $\mathbf{h}$ & $\mathbf{k}$ & $\ell$ & $\sin ^{2} \theta$ obs & $\sin ^{2} \theta_{\text {caic }}$ & $F_{\text {obs }}$ & $d(h k l)$ \\
\hline 5 & 5 & 2 & $i$ & 0.0733 & 0.0736 & 275 & 2.8416 \\
\hline 3 & 6 & $=$ & $\bar{z}$ & 0.0792 & 0.0795 & 204 & $\therefore .7336$ \\
\hline$<<$ & & & & unidentisied & inne & & \\
\hline$\vdots$ & 2 & 2 & 2 & 0.0815 & $0.08 I \varepsilon$ & 225 & 2.6956 \\
\hline 4 & $\left\{\begin{array}{l}7 \\
0\end{array}\right.$ & $i$ & $\overline{1}$ & 0.0827 & $\begin{array}{l}0.0826 \\
0.0831\end{array}$ & $\left.\begin{array}{c}119 \\
354\end{array}\right\}$ & 2.6760 \\
\hline$\vdots$ & $\left\{\begin{array}{l}2 \\
4\end{array}\right\}$ & $\frac{2}{2}$ & $\frac{I}{\Xi}$ & 0.0841 & $\begin{array}{l}0.0845 \\
0.0845\end{array}$ & $\left.\begin{array}{l}40 \\
45\end{array}\right\}$ & 2.6528 \\
\hline 4 & 7 & 2 & $\bar{\xi}$ & 0.0851 & 0.0854 & 254 & 2.6377 \\
\hline$?$ & 6 & 2 & $\overline{\bar{z}}$ & $0.0 \$ 36$ & 0.0937 & 98 & 2.5156 \\
\hline$<i$ & 7 & 2 & 0 & 0.0967 & 0.0968 & 55 & 2.4753 \\
\hline$<2$ & 3 & $i$ & 3 & 0.0978 & 0.0982 & 45 & 2.4605 \\
\hline 2 & 1 & 3 & $\dot{z}$ & 0.1006 & 0.1008 & 60 & 2.4268 \\
\hline : & $\leqslant 1$ & $z$ & $\equiv$ & 0.2027 & 0.2030 & $z$ & $\therefore .4017$ \\
\hline$=$ & $\left\{\begin{array}{l}6 \\
1 \\
\vdots \\
\vdots\end{array}\right.$ & $\begin{array}{l}20 \\
x: \\
\vdots \\
\vdots\end{array}$ & $\begin{array}{l}0 \\
\vdots \\
= \\
-\end{array}$ & 0.2003 & $\begin{array}{l}0 . i 062 \\
0.205 z \\
i .2006 \\
0.2065\end{array}$ & \begin{aligned} 20 & $\vdots \\
5 & \vdots \\
5 & \vdots \\
$\hdashline & 2\end{aligned} \mid & $\therefore 3600$ \\
\hline$z$ & $\left\{\begin{array}{l}z \\
\vdots\end{array}\right.$ & $\begin{array}{l}\vdots \\
z\end{array}$ & $\bar{\Sigma}$ & 0.1090 & $\begin{array}{l}0.1093 \\
0.2098\end{array}$ & $\left.\begin{array}{c}-40 \\
57\end{array}\right\}$ & $2 \cdot 3312$ \\
\hline 2 & $\left\{\begin{array}{l}2 \\
3 \\
z\end{array}\right.$ & $\begin{array}{ll}0 & 1 \\
3 & 0 \\
0 & 0\end{array}$ & $\frac{4}{5}$ & 0.1120 & $\begin{array}{l}0.1122 \\
0.1124 \\
0.1124\end{array}$ & $\left.\begin{array}{r}272 \\
29 \\
89\end{array}\right\}$ & 2.2996 \\
\hline 2 & 4 & 2 & 2 & 0.1235 & 0.2139 & 136 & 2.2842 \\
\hline is & $\left\{\begin{array}{l}x \\
5\end{array}\right.$ & $\begin{array}{l}2 \\
2 \\
2\end{array}$ & $\begin{array}{l}\bar{\Sigma} \\
\bar{\Xi}\end{array}$ & 0.1149 & $\begin{array}{l}0.2148 \\
0.2152\end{array}$ & $\left.\begin{array}{r}60 \\
283\end{array}\right\}$ & 2.2703 \\
\hline$<i$ & 6 & 0 & $\Sigma$ & 0.1167 & 0.1172 & 59 & 2.2526 \\
\hline$<1$ & & & & unidentified & Iine & & \\
\hline 3 & 7 & 13 & i & 0.1208 & 0.1213 & 287 & 2.2141 \\
\hline 3 & $\left\{\begin{array}{l}6 \\
g\end{array}\right.$ & $\begin{array}{ll}2 & 1 \\
1 & \end{array}$ & $\frac{\pi}{\bar{z}}$ & 0.1225 & $\begin{array}{l}0.1228 \\
0.1239\end{array}$ & $\left.\begin{array}{r}158 \\
50\end{array}\right\}$ & 2.1986 \\
\hline 2 & 9 & $i \overline{3}$ & $\overline{3}$ & 0.1247 & 0.1250 & iI4 & 2.1795 \\
\hline 2 & $\left\{\begin{array}{l}\xi \\
0\end{array}\right.$ & $\begin{array}{l}3 \\
2\end{array}$ & i & 0.1258 & $\begin{array}{l}0.1250 \\
0.1260\end{array}$ & $\left.\begin{array}{r}98 \\
108\end{array}\right\}$ & 2.1695 \\
\hline$\overline{3}$ & $I$ & ¿引 & $\overline{5}$ & 0.1283 & 0.1295 & $25 I$ & 2.2485 \\
\hline$z$ & $?$ & $1 \equiv$ & $\equiv$ & 0.1299 & 0.1297 & 64 & 2.1351 \\
\hline$T$ & $\left\{\begin{array}{l}8 \\
5\end{array}\right.$ & $\begin{array}{l}2 \\
3\end{array}$ & $\overline{\overline{2}}$ & $0 \frac{1}{3} 314$ & $\begin{array}{l}0.1318 \\
0.1318\end{array}$ & $\left.\begin{array}{l}325 \\
274\end{array}\right\}$ & $2.123 I$ \\
\hline
\end{tabular}




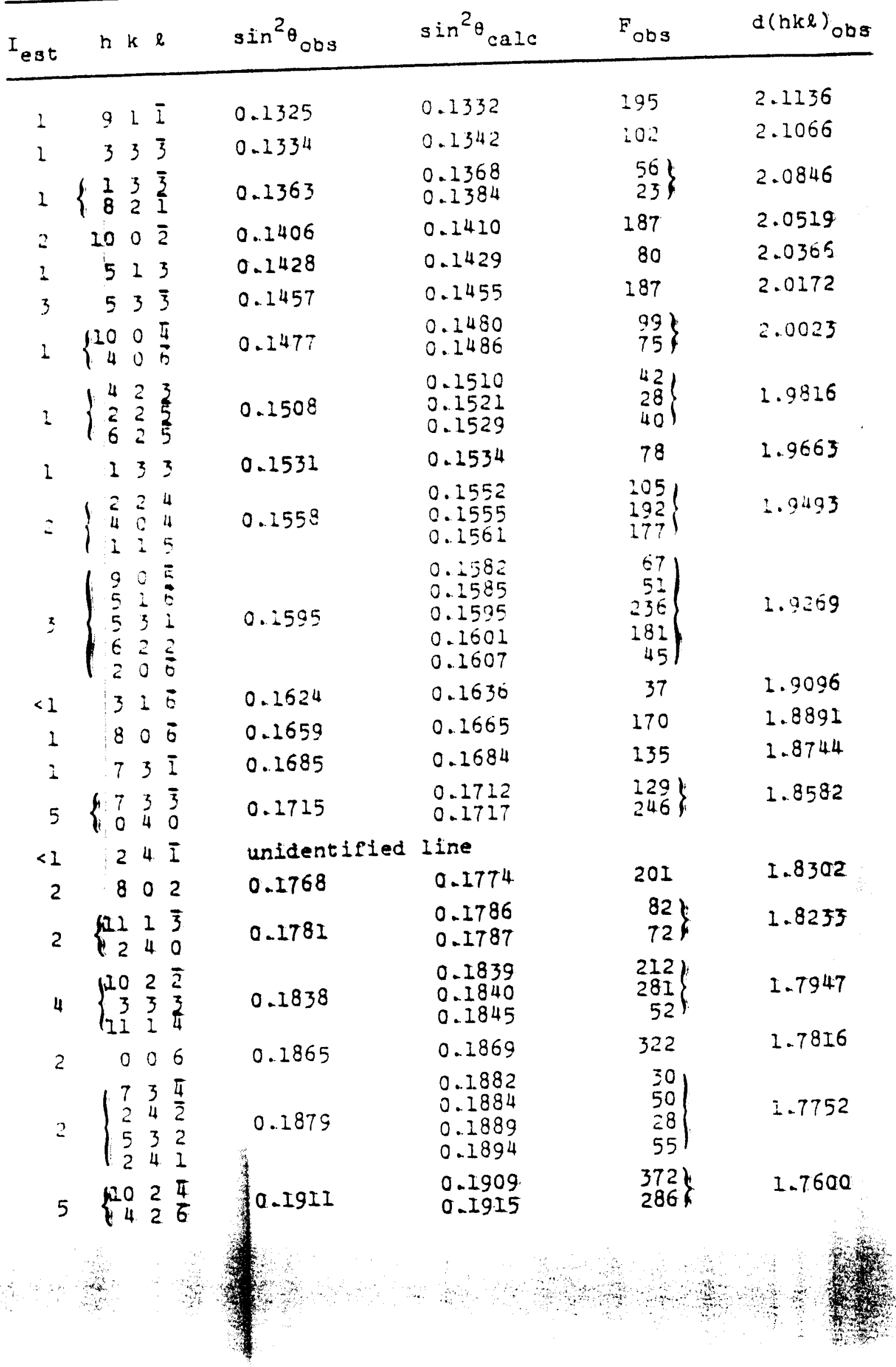




\begin{tabular}{|c|c|c|c|c|c|c|c|}
\hline Iest & $n$ & $k$ & $\ell$ & $\sin ^{2} \theta$ obs & $\sin ^{2} \theta \operatorname{calc}$ & Eqos & $d($ hkl $)$ obs \\
\hline 3 & 6 & 2 & & 0.1932 & 0.1934 & 240 & $1.7500^{\circ}$ \\
\hline 4 & $\mid \begin{array}{l}3 \\
1 \\
4 \\
4\end{array}$ & $\begin{array}{l}1 \\
1 \\
2 \\
4\end{array}$ & $\begin{array}{l}\frac{5}{1} \\
\frac{4}{2}\end{array}$ & $0 . \frac{7}{7} 81$ & $\begin{array}{l}0.1979 \\
0.1980 \\
0.1984 \\
0.1984\end{array}$ & $\begin{array}{r}106 \\
51 \\
238 \\
72\end{array} \mid$ & 1.7290 \\
\hline 6 & $\left\{\begin{array}{r}3 \\
12 \\
5 \\
7\end{array}\right.$ & $\begin{array}{l}3 \\
1 \\
3 \\
1\end{array}$ & $\begin{array}{l}\frac{5}{5} \\
\frac{5}{5} \\
\frac{5}{3}\end{array}$ & 0.2010 & $\begin{array}{l}0.2007 \\
0.2008 \\
0.20 I I \\
0.20 I 6\end{array}$ & $\left.\begin{array}{l}344 \\
155 \\
268 \\
239\end{array}\right)$ & \\
\hline 2 & 12 & 0 & 4 & 0.3029 & 0.2033 & 38 & \\
\hline 2 & $\left\{\begin{array}{r}7 \\
12\end{array}\right.$ & $\begin{array}{l}3 \\
0\end{array}$ & $\frac{1}{2}$ & $0 . \frac{3}{9} 70$ & $\begin{array}{l}0.2072 \\
0.2072\end{array}$ & $210\}$ & 1.6913 \\
\hline 1 & $\left\{\begin{array}{l}2 \\
9\end{array}\right.$ & $\begin{array}{l}4 \\
3\end{array}$ & $\frac{2}{3}$ & 0.2102 & $\begin{array}{l}0.2103 \\
0.2108\end{array}$ & $\left.\begin{array}{r}122 \\
75\end{array}\right\}$ & $1.67: 4$ \\
\hline 1 & $\left\{\begin{array}{l}5 \\
6\end{array}\right.$ & $\begin{array}{l}1 \\
0\end{array}$ & $\begin{array}{l}\overline{7} \\
4\end{array}$ & 0.2125 & $\begin{array}{l}0.2122 \\
0.2227\end{array}$ & $\begin{array}{l}116 \\
289\end{array}$ & $1.06: 2$ \\
\hline$z$ & 7 & 1 & $\bar{i}$ & 0.2154 & 2.2150 & $=0 \mathrm{r}$ & 1.053 \\
\hline 3 & $\left\{\begin{array}{r}10 \\
9\end{array}\right.$ & $\frac{2}{3}$ & $\frac{0}{2}$ & 0.2186 & $\begin{array}{l}0.2: 95 \\
0.2242\end{array}$ & $\left.\begin{array}{l}180 \\
220 \\
4\end{array}\right\}$ & $\therefore=0+3 i$ \\
\hline 3 & $\left\{\begin{array}{l}6 \\
3\end{array}\right.$ & $\begin{array}{l}4 \\
1\end{array}$ & $\frac{\bar{z}}{\bar{i}}$ & 0.2224 & $\begin{array}{l}0.2225 \\
0.2228\end{array}$ & $\begin{array}{l}18 \\
13\end{array}$ & $\therefore .0: 25$ \\
\hline 1 & 2 & 0 & 6 & 0.2267 & 0.2272 & 183 & 1.0161 \\
\hline$<<1$ & 0 & 2 & 6 & 0.2297 & 0.2298 & 82 & 1.0055 \\
\hline 2 & 9 & 1 & $\overline{7}$ & 0.2326 & 0.2330 & 217 & 1.5953 \\
\hline 3 & 6 & 4 & 0 & 0.2350 & 0.2549 & 213 & 1.5871 \\
\hline 2 & $\left\{\begin{array}{r}10 \\
2\end{array}\right.$ & $\begin{array}{l}2 \\
4\end{array}$ & $\frac{\overline{6}}{4}$ & 0.2395 & $\begin{array}{l}0.2394 \\
0.2396\end{array}$ & $\left.\begin{array}{l}I 77 \\
I 77\end{array}\right\}$ & 1.5723 \\
\hline 1 & $\mid \begin{array}{r}4 \\
12 \\
9 \\
6\end{array}$ & $\begin{array}{l}4 \\
2 \\
3 \\
2\end{array}$ & $\begin{array}{l}\frac{2}{3} \\
\frac{5}{7} \\
\frac{2}{7}\end{array}$ & 0.2433 & $\begin{array}{l}0.2427 \\
0.2429 \\
0.2441 \\
0.2443\end{array}$ & $\left.\begin{array}{l}43 \\
45 \\
39 \\
39\end{array}\right)$ & 1.5598 \\
\hline 4 & $\left\{\begin{array}{l}I 3 \\
I 3 \\
I\end{array}\right.$ & $\begin{array}{l}I \\
I \\
I\end{array}$ & $\frac{5}{\frac{4}{7}}$ & 0.2457 & $\begin{array}{l}0.2463 \\
0.2466 \\
0.2475\end{array}$ & $\left.\begin{array}{r}259 \\
42 \\
94\end{array}\right\}$ & 1.5524 \\
\hline 2 & 12 & 2 & $\bar{z}$ & 0.2494 & 0.2501 & 186 & 1.5406 \\
\hline 2 & $\left\{\begin{array}{r}6 \\
10 \\
12 \\
7 \\
5\end{array}\right.$ & $\begin{array}{l}4 \\
0 \\
0 \\
3 \\
1\end{array}$ & $\begin{array}{l}5 \\
2 \\
0 \\
6 \\
5\end{array}$ & 0.2513 & $\begin{array}{l}0.25 I 5 \\
0.25 I 7 \\
0.2529 \\
0.2533 \\
0.2536\end{array}$ & $\left.\begin{array}{r}168 \\
52 \\
32 \\
42 \\
124\end{array}\right)$ & 1.5350 \\
\hline
\end{tabular}




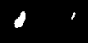

\begin{tabular}{|c|c|c|c|c|c|c|c|}
\hline$I_{-35}$ & $n$ & K & 2 & $\sin ^{2} \theta$ obs & $\sin ^{2} \theta \operatorname{caIc}$ & Fobs & $d($ hike $)$ obs \\
\hline 2 & 6 & 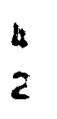 & + & 0.2546 & $\begin{array}{l}0.2549 \\
0.2556\end{array}$ & $\left.\begin{array}{l}203 \\
154\end{array}\right\}$ & $I .525 I$ \\
\hline$<I$ & 3 & $\begin{array}{l}1 \\
1\end{array}$ & $\equiv$ & 0.2587 & $\begin{array}{l}0.2574 \\
0.2598\end{array}$ & $\left.\begin{array}{l}I 07 \\
I 44\end{array}\right\}$ & \pm-5127 \\
\hline$<$ & & & & unidentified I & IIne & & \\
\hline : & $\begin{array}{l}\dot{3} \\
\vdots \\
\vdots\end{array}$ & $\begin{array}{l}4 \\
0\end{array}$ & $\begin{array}{l}\bar{\vdots} \\
\bar{\vdots}\end{array}$ & 0.2616 & $\begin{array}{l}0.2505 \\
0.2627\end{array}$ & $\left.\sum I I\right\}$ & 2.5043 \\
\hline$=$ & $\vdots$ & 2 & $\vdots$ & 0.2639 & $\begin{array}{l}0.2644 \\
0.2645\end{array}$ & $\left.\begin{array}{c}I g G \\
g I\end{array}\right\}$ & $L .497 T$ \\
\hline$\therefore:$ & $\begin{array}{r}5 \\
3 \\
32 \\
3\end{array}$ & $\begin{array}{l}4 \\
0 \\
2 \\
3\end{array}$ & $\begin{array}{l}\bar{\vdots} \\
\vdots \\
\vdots \\
\vdots\end{array}$ & 0.2670 & $\begin{array}{l}0.2671 \\
0.2676 \\
0.2678 \\
0.2589\end{array}$ & $\left.\begin{array}{r}62 \\
125 \\
97 \\
146\end{array}\right)$ & $I-489 I$ \\
\hline$\vdots$ & $\therefore$ & 2 & $\Xi$ & 2.2699 & $\begin{array}{l}0.2701 \\
0.2703\end{array}$ & $\left.\begin{array}{l}284 \\
296\end{array}\right\}$ & \pm-4820 \\
\hline 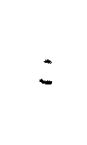 & $\stackrel{-}{2}$ & $i$ & $\bar{\vdots}$ & 0.2722 & $\begin{array}{l}0.2723 \\
0.274 \\
2.2744\end{array}$ & $\left.\begin{array}{r}1 \pm g \\
3 \pm \\
9 \pm\end{array}\right\}$ & $I \cdot-4 T+T$ \\
\hline$\vdots$ & $\frac{5}{3}$ & $\begin{array}{l}i \\
5 \\
i\end{array}$ & $\begin{array}{l}\bar{\vdots} \\
\vdots \\
\vdots\end{array}$ & 0.2775 & $\begin{array}{l}0.2767 \\
0.2780 \\
0.2785 \\
0.2796\end{array}$ & $\begin{array}{r}97 \\
278 \\
45 \\
174\end{array} \mid$ & \pm .4606 \\
\hline : & $\begin{array}{l}2 \\
5 \\
5\end{array}$ & $\begin{array}{l}2 \\
5 \\
0\end{array}$ & $\begin{array}{l}\vdots \\
\vdots \\
\vdots\end{array}$ & 2.2805 & $\begin{array}{l}0.2808 \\
0.2909 \\
0.2815\end{array}$ & $\left.\begin{array}{r}37 \\
I I g \\
I 32\end{array}\right\}$ & 2.4525 \\
\hline 4 & $\left\{\begin{array}{r}-2 \\
3 \\
1 \\
2 \\
2 \\
8 \\
3 \\
8\end{array}\right.$ & $\begin{array}{l}2 \\
3 \\
3 \\
8 \\
0 \\
5 \\
4\end{array}$ & $\begin{array}{l}5 \\
5 \\
5 \\
5 \\
4 \\
4 \\
0 \\
0\end{array}$ & 0.2836 & $\begin{array}{l}0.2834 \\
0.2837 \\
0.2838 \\
0.2839 \\
0.2840 \\
0.284 I \\
0.284 I\end{array}$ & $\begin{array}{r}15 I \\
2 I I \\
255 \\
64 \\
165 \\
2 I I \\
46\end{array}$ & $I .4449$ \\
\hline 4 & $\left\{\begin{array}{l}1 \\
20 \\
-\frac{1}{4}\end{array}\right.$ & $\begin{array}{l}1 \\
0 \\
3 \\
0 \\
3\end{array}$ & $\begin{array}{l}\frac{T}{3} \\
\vdots \\
\frac{2}{2} \\
\vdots\end{array}$ & 0.2864 & $\begin{array}{l}a-2863 \\
a-2865 \\
0.2866 \\
a-2874 \\
a-2875\end{array}$ & $\begin{array}{r}I 8 d \\
75 \\
\Sigma I 7 \\
228 \\
I \Sigma 7\end{array}$ & $I-4376$ \\
\hline$z$ & $\left\{\begin{array}{l}-0 \\
2\end{array}\right.$ & $\begin{array}{l}2 \\
0\end{array}$ & 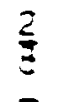 & 0.2446 & $\begin{array}{l}0.2946 \\
0.2950\end{array}$ & $\begin{array}{l}269 \\
144\end{array}$ & $I-4 I 75$ \\
\hline 2 & $\left\{\begin{array}{l}5 \\
24\end{array}\right.$ & $\begin{array}{l}3 \\
0\end{array}$ & $\overline{\bar{\gamma}}$ & 0.2976 & $\begin{array}{l}0.2980 \\
a .2986\end{array}$ & 565\% & $I-4 I 03$ \\
\hline
\end{tabular}

